\title{
Pneumococcal Disease Outbreak at a State Prison, Alabama, USA, September 1-October 10, 2018
}

\author{
Guillermo V. Sanchez, Constance L. Bourne, Sherri L. Davidson, Mark Ellis, Leora R. Feldstein, \\ Katherine Fay, Nicole E. Brown, Evelyn F. Geeter, Lytasha L. Foster, Charlotte Gilmore, \\ Mary G. Mclntyre, Burnestine Taylor, Srinivasan Velusamy, Sopio Chochua, Almea M. Matanock
}

A pneumococcal disease outbreak caused by Streptococcus pneumoniae serotype $12 \mathrm{~F}$ occurred in a state prison in Alabama, USA. Among 1,276 inmates, 40 cases were identified (3 confirmed, 2 probable, 35 suspected). Close living quarters, substance use, and underlying conditions likely contributed to disease risk. Prophylaxis for close contacts included azithromycin and 23-valent pneumococcal polysaccharide vaccine.

Streptococcus pneumoniae (pneumococcus) causes a $\mathcal{S}$ spectrum of disease ranging from mild respiratory infections to severe disease, including meningitis, sepsis, and pneumonia (1). Invasive pneumococcal disease (IPD) occurs when pneumococcus invades normally sterile sites. Pneumococcus is transmitted person-to-person primarily through respiratory droplets and is a leading cause of vaccine-preventable illness and death (2). Pneumococcal colonization is a precursor to disease but does not always result in disease (3). Pneumococcal conjugate vaccine (PCV) is highly effective in preventing pneumonia in adults (4), and pneumococcal disease incidence has declined since the introduction of PCV (5). IPD outbreaks are rare but can occur in settings with close personto-person contact, such as homeless shelters (6) and healthcare facilities, in which underlying conditions can increase disease risk (7).

On September 19, 2018, the Alabama Department of Public Health (Montgomery, AL) was notified

Author affiliations: Centers for Disease Control and Prevention, Atlanta, Georgia, USA (G.V. Sanchez, L.R. Feldstein, K. Fay,

N.E. Brown, S. Velusamy, S. Chochua, A.M. Matanock); Alabama Department of Public Health, Montgomery, Alabama, USA

(G.V. Sanchez, C.L. Bourne, S.L. Davidson, M. Ellis, E.F. Geeter, L.L. Foster, C. Gilmore, M.G. McIntyre, B. Taylor)

DOI: https://doi.org/10.3201/eid2707.203678 of an IPD case after identification of S. pneumoniae in a blood culture from an ill patient incarcerated at a state prison. On September 24, a second case of IPD was reported in another inmate who received a diagnosis of meningitis and sepsis and died that morning. We investigated this outbreak to determine its extent, identify cases among staff and inmates, and recommend prophylactic measures to reduce spread.

\section{The Study}

At the time of the outbreak, facility A, a medium-security state prison, housed 1,276 male inmates across 6 dormitories (original capacity 650 inmates; 2018 reported capacity of 1,650 inmates) (8,9). Each dormitory contained multiple large rooms with 4-6 rows of beds for 190-255 inmates. Group activities allowed mixing of inmates from different dorms until the outbreak was recognized; activities were suspended around September 26. A clinic within facility A with a 52-member staff, including 2 nurse practitioners and a physician, provided services to inmates through self or employee referral.

A suspected case was defined as respiratory or meningeal symptoms consistent with pneumococcal disease in an incarcerated person or a person in prolonged or close contact with anyone incarcerated at facility A during September 1-October 10, 2018 (Appendix, https://wwwnc.cdc.gov/EID/ article/27/7/20-3678-App1.pdf). Probable cases were defined as suspected cases with radiographicconfirmed pneumonia, clinical sepsis, or cerebrospinal fluid analysis suggestive of bacterial meningitis with unknown etiology. Confirmed cases were

${ }^{1}$ Preliminary results from this study were presented at the Centers for Disease Control and Prevention 2019 Epidemic Intelligence Service conference, April 29-May 2, 2019, Atlanta, Georgia, USA. 
defined as suspected cases with S. pneumoniae isolation or positive urinary antigen test.

We conducted retrospective case finding among inmates who were seen in the clinic during September 1-September 29 and prospective surveillance during September 30-October 10. Cases in which inmates reported respiratory illness, altered mental status, headache, or fever and those without a listed chief complaint were flagged for medical chart review. Inmates whose medical records indicated signs or symptoms of pneumococcal disease were interviewed by using a standardized questionnaire to identify clinical characteristics, risk factors, and epidemiologic links with confirmed cases. For prospective surveillance, we screened all inmates who experienced respiratory symptoms for pneumococcus and influenza by using nasopharyngeal swabs. We tested influenza-negative swab specimens for other respiratory pathogens by using the BioFire FilmArray Respiratory Panel (bioMérieux, https://www.biomerieux.com). We performed pneumococcal serotyping on nasopharyngeal swab specimens from which S. pneumoniae was isolated. We calculated attack rates by dividing the number of identified cases by the at-risk population (i.e., all dormitory residents). Specimen culture and antibiotic susceptibility testing was performed at laboratories in the hospitals in which patients received care and confirmed at the Centers for Disease Control and Prevention. Sterile body fluids and nasopharyngeal swab specimens were sent to Centers for Disease Control and Prevention for species detection, confirmation, and serotyping by using real-time reverse transcription PCR. Bacterial serotyping and whole-genome sequencing (WGS) were performed on pneumococcal isolates collected from confirmed cases. We analyzed single-nucleotide polymorphisms identified through WGS to verify temporal relatedness of the isolates.

Through retrospective case finding, 96 medical chart reviews and 52 inmate interviews identified 40 cases ( 3 confirmed, 2 probable, and 35 suspected;

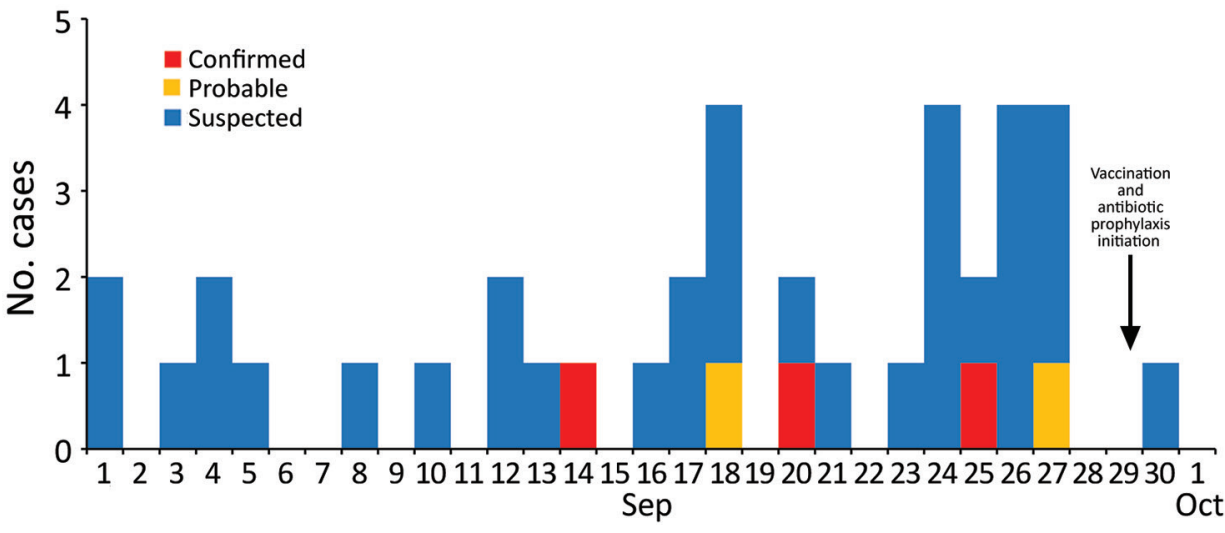

Figure) for attack rates of $3 \%(40 / 1,276)$ within facility A and 5\% (14/255) within dormitory X. All confirmed cases occurred in inmates living in dormitory $X$. Suspected cases were identified among inmates in all 6 dormitories. No pattern of temporal spread was observed among dormitories. Of suspected cases, $26 \%$ (9/35) reported lower respiratory symptoms (chest pain, shortness of breath, or fever with cough) (Table 1). Underlying conditions for which 23-valent pneumococcal polysaccharide vaccine (PPSV23) is routinely recommended (excluding smoking tobacco) (10) were reported in $2 / 5(40 \%)$ confirmed or probable cases and 6/35 (17\%) suspected cases. In 3/35 $(9 \%)$ suspected cases, patients were immunocompromised. Of the $28 / 40$ (70\%) patients who reported smoking cigarettes, half $(14 / 28,50 \%)$ reported sharing cigarettes.

Blood $(n=5)$ or cerebrospinal fluid $(n=4)$ specimens were collected from 5 patients. Three S. pneumoniae isolates were identified from 3 patients ( 2 from cerebrospinal fluid, 1 from blood); all 3 were serotype 12F. WGS demonstrated a $2-5$ single-nucleotide polymorphism difference among isolates, indicating all confirmed cases were closely related. Antimicrobial susceptibility testing confirmed isolate susceptibility.

During prospective surveillance, we collected nasopharyngeal swab specimens from 2 inmates; $S$. infantis serotype 13 was detected in 1 specimen and adenovirus only in the other. No additional cases were identified. Prophylaxis with PPSV23, which protects against serotype $12 \mathrm{~F}$, and 2 doses of azithromycin were offered to inmates and close contacts living in or assigned to dormitory $\mathrm{X}$, clinic staff, and prison employees (Table 2).

\section{Conclusions}

This investigation highlights the outbreak potential of S. pneumoniae and demonstrates that correctional facilities remain at risk for pneumococcal outbreaks after PCV introduction in the United States. The last
Figure. Epidemic curve of identified cases among inmates in study of pneumococcal disease outbreak at a state prison, Alabama, USA, September 1-October 10, 2018. Date of first symptom onset is shown. Healthcare unit visit date was used when symptom onset date was not known. Two suspected cases without a clear onset date were excluded from this graph. 
Table 1. Patient demographics, signs, symptoms, and Streptococcus pneumoniae risk factors by case classification in study of pneumococcal disease outbreak at a state prison, Alabama, USA, September 1-October 10, 2018*

\begin{tabular}{|c|c|c|c|}
\hline Demographics & Confirmed cases, $n=3$ & Probable cases, $n=2$ & Suspected cases, $n=35$ \\
\hline Median age, y (range) & $46(44-61)$ & $42(26-58)$ & $39(23-64)$ \\
\hline \multicolumn{4}{|l|}{ Race } \\
\hline Black & $3(100)$ & 0 & $14(40)$ \\
\hline White & 0 & $2(100)$ & $13(37)$ \\
\hline Unknown & 0 & 0 & $8(23)$ \\
\hline \multicolumn{4}{|l|}{ Signs or symptoms } \\
\hline Fever & $2(67)$ & $1(50)$ & $15(43)$ \\
\hline Cough & $2(67)$ & $1(50)$ & $19(54)$ \\
\hline Shortness of breath & $1(33)$ & $2(100)$ & $4(11)$ \\
\hline Chest pain & $1(33)$ & $1(50)$ & $6(17)$ \\
\hline Headache & $1(33)$ & 0 & $11(31)$ \\
\hline Neck stiffness & $2(67)$ & 0 & $1(3)$ \\
\hline Altered mental status & $3(100)$ & $1(50)$ & 0 \\
\hline Congestion & 0 & 0 & $6(17)$ \\
\hline \multicolumn{4}{|l|}{ Clinical features and risk factors } \\
\hline Immunocompromising condition† & 0 & 0 & $3(9)$ \\
\hline Chronic medical condition $\ddagger$ & $1(33)$ & $1(50)$ & $6(17)$ \\
\hline Substance use: cigarettes, alcohol, or illicit drugs & $2(67)$ & 0 & $27(77)$ \\
\hline \multicolumn{4}{|l|}{ Assigned housing } \\
\hline Dormitory $\mathrm{X}$ & $3(100)$ & 0 & $11(31)$ \\
\hline Dormitory $Y$ & 0 & $1(50)$ & $8(23)$ \\
\hline Other dormitories: $\mathrm{U}, \mathrm{V}, \mathrm{W}, \mathrm{Z}$ & 0 & 0 & $16(46)$ \\
\hline Healthcare unit & 0 & $1(50)$ & 0 \\
\hline
\end{tabular}

*Values are no. (\%) except as indicated. Table includes composite statistics from both inmate interviews and medical chart abstractions. All identified cases occurred in inmates; no cases were identified among staff members.

†Includes immune suppression, chronic renal failure, HIV, solid organ transplant, asplenia, sickle cell disease and other hemoglobinopathies, and malignancy excluding skin cancer (Centers for Disease Control and Prevention Adult Vaccine Schedule, https://www.cdc.gov/vaccines/acip).

†Includes chronic heart, liver, kidney, lung diseases, and diabetes mellitus; excludes documented substance abuse, Hepatitis $\mathrm{C}$ infection, and chronic osteomyelitis (Centers for Disease Control and Prevention Adult Vaccine Schedule, https://www.cdc.gov/vaccines/acip).

documented pneumococcal disease outbreak at a US correctional facility also involved serotype $12 \mathrm{~F}$ and occurred in 1989 in a crowded Texas jail, in which 46 inmates experienced pneumonia, meningitis, or sepsis over a 4 -week period (11). Unlike jails, which have an average detention length of $<2$ months, prisons have lower rates of inmate turnover because they are designed for long-term incarceration; the average detention among US state prisoners in 2016 was 2.6 years (12). However, jails and prisons are similar in that they share risk factors for outbreaks, such as potential overcrowding and increased medical and behavioral risk factors for communicable diseases (13). Decreases in pneumococcal disease have been observed during outbreaks after administering prophylactic antibiotics and PPSV23 to high-risk persons $(6,7,11,14)$. Risk for pneumococcal disease outbreaks in prisons can be minimized by offering inmates vaccinations per Advisory Committee on Immunization Practices recommendations, which recommend PPSV23 for persons with chronic heart, liver, or lung disease (15). Risk can be further reduced by minimizing inmate crowding, eliminating indoor smoking, and ensuring adequate ventilation (11).

Table 2. Administration of pneumococcal polysaccharide vaccine and antibiotic prophylaxis to reduce Streptococcus pneumoniae transmission in prison inmates during a pneumococcal disease outbreak, Alabama, USA, September 29-October 10, 2018*

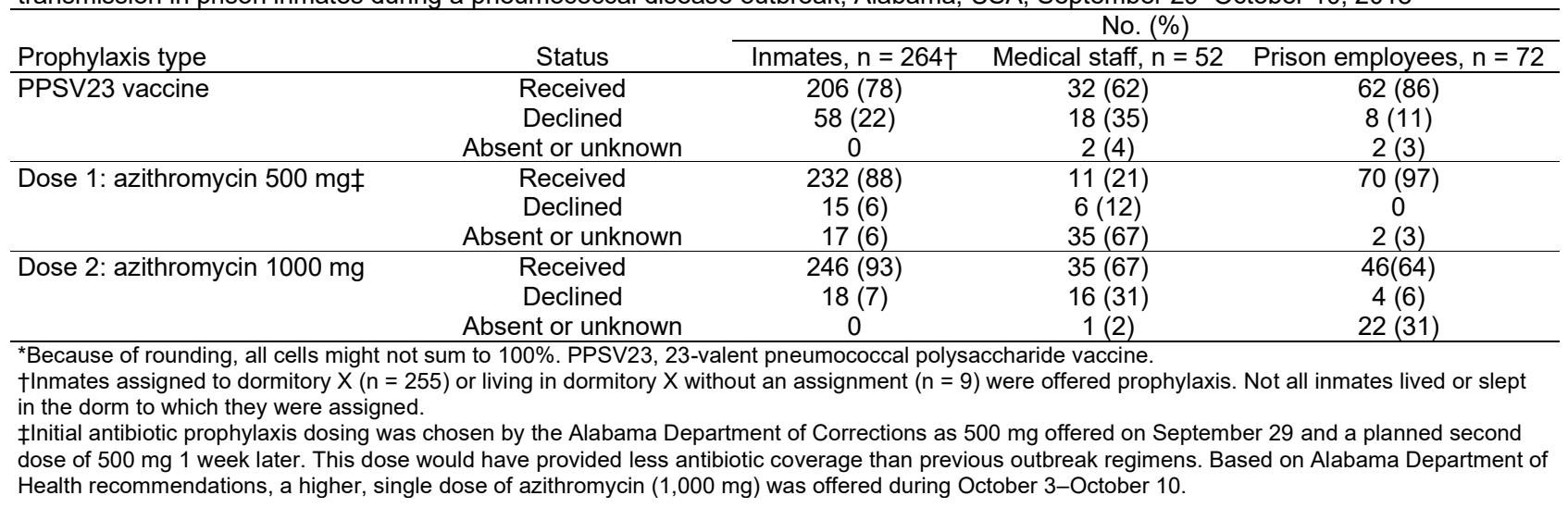


One limitation of this study was that risk factor information was collected by self-reporting and might be underestimated. Disease etiology was not confirmed for most cases because few patients had confirmatory laboratory testing for respiratory pathogens. Dormitories were not assessed for space, capacity, or ventilation.

Pneumococcal colonization among inmates could not be widely assessed. We observed decreases in pneumococcal disease after prophylaxis administration, but we cannot determine the direct impact of prophylaxis since $S$. pneumoniae serotype $12 \mathrm{~F}$ carriage was not measured. Pneumococcal carriage studies among incarcerated populations could further our understanding of pneumococcal disease in correctional facilities.

In our outbreak investigation of pneumococcal disease in a state prison, we observed decreases in disease after prophylaxis with PPSV23 and azithromycin. Increased pneumococcal disease risk might have resulted from close living quarters, substance use, and underlying conditions. Improved pneumococcal disease surveillance and proactive vaccination of at-risk inmates in accordance with Advisory Committee on Immunization Practices recommendations might mitigate risk for and scale of future outbreaks.

\section{Acknowledgments}

We thank the Alabama Department of Corrections for collaboration during this investigation.

\section{About the Author}

Mr. Sanchez is an Epidemic Intelligence Service officer with the National Center for Emerging and Zoonotic Infectious Diseases, Centers for Disease Control and Prevention, assigned to the Alabama Department of Public Health. His research interests include antibiotic resistance, antibiotic stewardship, and healthcareassociated infections.

\section{References}

1. Simell B, Auranen K, Käyhty H, Goldblatt D, Dagan R, O'Brien KL; Pneumococcal Carriage Group. The fundamental link between pneumococcal carriage and disease. Expert Rev Vaccines. 2012;11:841-55. https://doi.org/10.1586/erv.12.53

2. Centers for Disease Control and Prevention. Pneumococcal disease: types of infection [cited 2019 Nov 14]. https:/ / www. cdc.gov/pneumococcal/about/infection-types.html
3. Bogaert D, De Groot R, Hermans PWM. Streptococcus pneumoniae colonisation: the key to pneumococcal disease. Lancet Infect Dis. 2004;4:144-54. https:/ / doi.org/10.1016/ S1473-3099(04)00938-7

4. Bonten MJM, Huijts SM, Bolkenbaas M, Webber C, Patterson S, Gault S, et al. Polysaccharide conjugate vaccine against pneumococcal pneumonia in adults. N Engl J Med. 2015;372:1114-25. https:/ / doi.org/10.1056/NEJMoa1408544

5. Shiri T, McCarthy ND, Petrou S. The impact of childhood pneumococcal vaccination on hospital admissions in England: a whole population observational study. BMC Infect Dis. 2019;19:510. https:/ / doi.org/10.1186/ s12879-019-4119-8

6. Romney MG, Hull MW, Gustafson R, Sandhu J, Champagne S, Wong T, et al. Large community outbreak of Streptococcus pneumoniae serotype 5 invasive infection in an impoverished, urban population. Clin Infect Dis. 2008;47:768-74. https:/ / doi.org/10.1086/591128

7. Fleming-Dutra K, Mbaeyi C, Link-Gelles R, Alexander N, Guh A, Forbes E, et al. Streptococcus pneumoniae serotype 15A in psychiatric unit, Rhode Island, USA, 2010-2011. Emerg Infect Dis. 2012;18:1889-93. https:/ / doi.org/10.3201/ eid1811.120454

8. Dunn JS, Thomas KT. Alabama Department of Corrections Monthly Statistical Report. September 2018 [cited 2020 Feb 5]. http:/ / www.doc.state.al.us/docs/MonthlyRpts/ 2018-09.pdf

9. Alabama Department of Corrections. Ventress Correctional Facility [cited 2019 Feb 20]. http:/ / www.doc.state.al.us/ facility? 1 loc $=38$

10. Centers for Disease Control and Prevention. Pneumococcal vaccine timing for adults [cited 2018 Oct 1]. https://www.cdc.gov/vaccines/vpd/pneumo/downloads/ pneumo-vaccine-timing.pdf

11. Hoge CW, Reichler MR, Dominguez EA, Bremer JC, Mastro TD, Hendricks KA, et al. An epidemic of pneumococcal disease in an overcrowded, inadequately ventilated jail. N Engl J Med. 1994;331:643-8. https:// doi.org/10.1056/NEJM199409083311004

12. Kaeble D. Time served in state prison, 2016. November 2018 [cited 2020 Feb 5]. https:/ / www.bjs.gov/content/pub/pdf/ tssp16.pdf

13. Binswanger IA, Krueger PM, Steiner JF. Prevalence of chronic medical conditions among jail and prison inmates in the USA compared with the general population. J Epidemiol Community Health. 2009;63:912-9. https:/ / doi.org/10.1136/ jech.2009.090662

14. Crum NF, Wallace MR, Lamb CR, Conlin AMS, Amundson $\mathrm{DE}$, Olson PE, et al. Halting a pneumococcal pneumonia outbreak among United States Marine Corps trainees. Am J Prev Med. 2003;25:107-11. https://doi.org/10.1016/ S0749-3797(03)00114-4

15. Centers for Disease Control and Prevention. Advisory Committee on Immunization Practices (ACIP) [cited 2019 Nov 14]. https://www.cdc.gov/vaccines/acip/index.html

Address for correspondence: Guillermo V. Sanchez, Centers for Disease Control and Prevention, 1600 Clifton Rd NE, Mailstop H16-3, Atlanta, GA 30329, USA; email: gsanchez@cdc.gov 